\title{
UNA CONSTRUCCIÓN DE LAS REGLAS DE CÁLCULO DE LOS NÚMEROS ENTEROS A PARTIR DE LA MANIPULACIÓN DE EXPRESIONES ALGEBRAICAS
}

\author{
Patricia Detzel, María Elena Ruiz y Lucas Colipe
}

\begin{abstract}
RESUMEN. En este artículo presentamos parte de un trabajo desarrollado en un proyecto de investigación, en el que abordamos, en forma conjunta investigadores y profesores de matemática de escuelas secundarias, la problemática de la enseñanza de los números enteros desde una perspectiva colaborativa. Dicha problemática era una preocupación de los profesores de matemática, debido a las dificultades que observaban en sus alumnos, en particular en relación a la manipulación de los signos en los cálculos. Para ello acordamos en conjunto, estudiar y adaptar una propuesta de enseñanza en la que se aborda la introducción de los números negativos conjuntamente con el álgebra. Transitamos un proceso de esclarecimiento que nos permitió cargar de sentido un recorrido, en el que surgen las reglas de cálculo para la suma, resta y multiplicación de sumandos y sustraendos apoyadas en los conocimientos de los números naturales, donde las expresiones algebraicas tuvieron un rol fundamental. Desarrollamos en este artículo, parte de los análisis y reflexiones que llevamos a cabo profesores e investigadores en ese proceso para la adaptación de dicha propuesta.
\end{abstract}

Palabras clave: números enteros, actividad matemática, ruptura entre aritmética y álgebra, enseñanza escuela secundaria.

Keywords: integers, mathematical activity, gap between algebra and arithmetic, secondary school teaching. 


\begin{abstract}
AвSTRACT. This article presents part of a research developed in collaboration, by math education researchers and teachers of mathematics at the secondary level. The work addresses the problems encountered by teachers when teaching integers, and the difficulties students had grasping the concept, particularly in reference to the sign manipulation needed to carry on computations. Because of this premise, we all decided to study and make adaptations to an instructional proposal focused on the presentation of the negative integers together with that of algebra. We navigated a process of clarification that gave room for us to follow a meaningful path where the rules of addition, subtraction, and multiplication can be developed based on the knowledge of natural numbers. The role of algebra was fundamental in following this path. This article provides the analysis and reflections of the researchers and math teachers while adapting the original instructional approach.
\end{abstract}

\title{
§1. Introducción
}

Este trabajo se enmarca en un proyecto de investigación que desarrollamos en la Universidad Nacional del Comahue (UNCo), en el que abordamos, en forma conjunta investigadores y profesores de matemática de escuelas secundarias, problemáticas de la enseñanza de la matemática desde una perspectiva colaborativa (Desgagné, Bednarz, Couture, y Lebuis, 2001; Bednarz, 2015, 2013, 1997). En particular, en este artículo nos centramos en una experiencia compartida con profesores que consistió en el estudio y adecuación a sus aulas, de una producción de investigación en didáctica de la matemática.

A partir de la demanda de los profesores, preocupados por dificultades que tienen los estudiantes en el transcurso de la escuela secundaria, en relación a la manipulación de los signos en los cálculos, acordamos abordar conjuntamente la problemática de la enseñanza de los números negativos, en los primeros años de la educación secundaria. Para ello nos basamos en el trabajo desarrollado por Eva Cid en su tesis doctoral (Cid, 2015), quien propone introducir los números negativos conjuntamente con el álgebra. Así, aceptamos involucrarnos en un proceso de readaptación de dicha propuesta para la enseñanza de los números enteros, cuya experiencia tuvo lugar desde 2013 hasta 2019 en escuelas de Río Negro y Neuquén.

En este sentido, (Perrin-Glorian, 2011; Perrin-Glorian y Baltar Bellemain, 2019), destacan la importancia de estudiar la adaptación de ingenierías didácticas ${ }^{1}$ a las condiciones ordinarias de enseñanza y a las necesidades de los docentes. Los espacios colectivos de trabajo entre docentes e investigadores, constituyen un escenario favorable para discutir con los docentes las cuestiones fundamentales que se ponen en juego en tales secuencias de enseñanza más que para difundir los productos acabados (Sadovsky, Itzcovich, Quaranta, Becerril, y García, 2016). En este caso, discutir la contextualización y descontextualización de los modelos

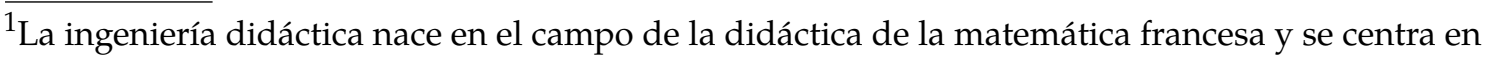
modelar situaciones de enseñanza, para así permitir el estudio de su funcionamiento en las aulas.
} 
matemáticos y los diferentes funcionamientos de algunos objetos en aritmética y en álgebra, entre otros, fueron asuntos importantes de esta propuesta para llevar adelante una posible gestión de las actividades en las clases.

En la propuesta (Cid y Ruiz-Munzón, 2011) que analizamos y estudiamos se construyen reglas de cálculo para sumar, restar y multiplicar los números enteros. Esta construcción toma como base conocimientos del cálculo aritmético que los estudiantes disponen y se propone, mediante rupturas, hacerlos evolucionar hacia nuevos conocimientos propios del álgebra. Según estas autoras (Cid y RuizMunzón, 2011), "la razón de ser" ${ }^{2}$ de los números negativos se encuentra en el cálculo algebraico, es decir, en la medida en que se avanza en la gestión de dichos cálculos, a partir de la manipulación de determinadas expresiones algebraicas, se gana en comprensión en la operatoria de los números enteros. Para ello, se parte de problemas del mundo sensible, que dan lugar a crear modelos que son expresiones algebraicas o programas de cálculos aritméticos (PCA) ${ }^{3}$, para luego centrar el estudio en el funcionamiento de los mismos. Además de crear el modelo, es importante aprender a trabajar con el mismo. El problema en contexto permite al estudiante "hacer" y controlar que lo que hace funciona, admite una resolución a partir de las acciones de añadir, quitar, ganar, perder, subir, bajar. Sin embargo, la producción de una expresión algebraica y el análisis del desarrollo de los cálculos involucrados en la misma, permitirá reemplazar estas acciones por las de sumar y restar. Es necesario entonces, un proceso de descontextualización, en el que esos modelos se tomen como objeto de estudio, dando posibilidad a que emerjan las reglas de cálculos entre sumandos y sustraendos que se constituirán en el soporte para la operatoria de los números enteros.

Por otro lado, se asocian conceptos matemáticos con problemas cuya resolución permite, a partir de saberes disponibles, elaborar relaciones que darán lugar a conocimientos nuevos. En nuestro caso, esos conocimientos nuevos que circularán en la clase, forman parte de la propuesta de enseñanza y son inéditos para el ámbito escolar. Los mismos y su vínculo con las actividades fueron ideados en el ámbito de una investigación (producto de una tesis doctoral) en la que no habíamos participado (docentes e investigadores de este grupo colaborativo). Entonces, comprender el andamiaje construido con los problemas en contexto, la manipulación de expresiones y el trabajo con sumandos y sustraendos, que permite conectar los saberes conocidos (los números naturales) y los que se desea

\footnotetext{
${ }^{2}$ La teoría antropológica de los didáctico (TAD) propone un paradigma didáctico de cuestionamiento del mundo que propugna una presentación funcional del saber. Esta manera de entender la enseñanza de las matemáticas convierte los procesos de modelización matemática, tanto externa como interna, en el eje que vertebra dicha enseñanza, proporcionando las razones de ser que dan sentido a los objetos matemáticos y poniendo de manifiesto las relaciones que existen entre ellos, en vez de presentarlos como objetos aislados (Cid, Muñoz-Escolano, y Ruiz-Munzón, 2020).

${ }^{3}$ (Chevallard, 2005) le da el nombre de programa de cálculo aritmético (PCA) a una cadena de operaciones aritméticas que se realizan a partir de los datos de un problema.
} 
enseñar (los números enteros), fue una cuestión a esclarecer. Tal reconstrucción de ideas conlleva a (re)diseñar un recorrido que asegure una coherencia entre los aprendizajes y lo que se quiere enseñar (Sadovsky y cols., 2016). Así, el proceso de adaptación compartido (investigadores y profesores) nos permitió avanzar en comprensión de ese andamiaje, involucrándonos en un recorrido de estudio para una construcción escolar de las operaciones suma, resta y producto de números enteros, apoyada en los conocimientos de los números naturales. Dicho recorrido está atravesado por rupturas entre concepciones y modos de calcular que viven en la aritmética y que evolucionan a nuevas concepciones y funcionamientos en el álgebra. En este texto compartimos parte de ese recorrido que permite evidenciar el potencial de expresiones algebraicas que surgen de los problemas, como objeto de estudio para construir reglas de cálculos de sumandos y sustraendos emergentes, las que a su vez permiten cargar de sentido a la operatoria de números enteros.

\section{§2. Acerca de la construcción de las reglas de cálculo para sumar}

Como lo mencionamos anteriormente, la razón de ser de los números negativos se encuentra en el álgebra. Es importante reconocer que, en la suma, una ruptura entre lo aritmético y lo algebraico, aparece con el cambio de significado de los signos $+\mathrm{y}-$. En aritmética tienen un significado binario, determinan operaciones, mientras que en álgebra pasan a tener otros significados como los de operador unario y predicativo (Detzel y Martinez, 2017).

Esto nos condujo a que, en los espacios de estudio compartidos con los docentes, tuviéramos que resignificar ideas que permitan construir lazos entre "sumas y restas de números naturales" con "una suma de enteros que no se escribe". Nos llevó así, a reconocer que en el procedimiento $9-5-2+5=9-2$ se está resolviendo una suma que no aparece: $9+(-5)+(-2)+(+5)$. Y por otro lado advertir que los significados de los signos + y - cambian según las decisiones que se toman en los cálculos. Por ejemplo, en la expresión $12-4-5+4$ si se hace $12-4-5+4=8-5+4=3+4=7$ los signos $+y-$ estarían funcionando como operadores binarios y se estarían resolviendo sumas y restas entre números naturales. Sin embargo, si se resuelve $12-4-5+4=12-5-4+4=12-5=7$ los signos + y - están funcionando como predicativo (signo que acompaña al número). En este caso se está resolviendo la suma de los términos $+12,-4,-5$ $\mathrm{y}+4$, donde el signo binario, que indica la suma entre ellos, no aparece. Es una suma no escrita la que habilita ese modo de hacer, la resta entre naturales no es conmutativa. Reconocer que en este cálculo están involucradas implícitamente las propiedades asociativa, conmutativa, existencia del neutro y de los opuestos, propias de una suma de los números enteros, es lo que posibilita entrar en un proceso de construcción de dicha operación. 
El tratamiento de expresiones tales como $4-10+3+10$ donde la sucesión de cálculos de sumas y restas como números naturales no es posible, dispone a iniciar un trabajo para facilitar resoluciones del tipo $4+3-10+10=4+3=7$, donde la suma de números enteros a nivel implícito es el saber involucrado. La evolución de un cálculo aritmético a un cálculo de tipo algebraico implica abordar los diferentes sentidos de los signos + y -, también lograr que las sumas y restas de naturales avancen transformándose en una suma implícita de enteros. Un modo de realizar ese proceso, según lo proponen (Cid, 2015; Cid y Bolea, 2010), es a partir de construir reglas de cálculos de sumandos y sustraendos usando expresiones algebraicas. Con estas reglas para simplificar expresiones, está presente la idea de suma como composición de traslaciones y los significados, operativo binario generalizado, de los signos + y - (Barrio y Petich, 2018). Es decir, operar con sumandos y sustraendos, permite que funcione la suma a nivel implícito de números enteros donde valen las propiedades conmutativa y asociativa (cuestión que se pierde si se piensa sólo en sumas y restas de números naturales). También se va estableciendo una representación horizontal por sobre la vertical.

2.1. Actividades para construir las reglas de cálculo para sumar. En un principio las actividades propuestas corresponden a problemas aritméticos contextualizados donde la falta de alguno de los datos necesarios para resolverlos, fuerza a la utilización de letras y de este modo la solución a un problema cambia de status, ya no es un número, sino que es una expresión algebraica. Será necesario, entonces, habilitar un contexto algebraico en el que el modelo $x-a+b$ posibilite cálculos que permitan gestionar el paso de expresiones del tipo $x-9+7$ a su forma reducida $x-2$.

Los problemas contextualizados del tipo:

- Ana lleva sus figuritas para jugar en la escuela. En el primer recreo pierde 9 y en el segundo recreo gana 7. ¿Con cuántas figuritas vuelve a su casa?

- El colectivo KoKo sale de Roca y viaja a Neuquén con cierta cantidad de pasajeros. En Allen bajan 15 y suben 12, luego en Cipolletti bajan 38 y suben 42. ¿Con cuántos pasajeros llega a Neuquén?

dan lugar a encontrar respuestas en el contexto. En el primer caso, una respuesta posible es: "llega con dos figuritas menos"; en el segundo caso una respuesta podría ser: "llega a Neuquén con un pasajero más".

Los problemas descontextualizados del tipo: 
- Un chico, al hacer los deberes, tiene que simplificar unas expresiones algebraicas. Lo hace de la siguiente manera:

(a) $d-7-4+10=d-3+10=d-13$.

(b) $27-q-8-8=27-q-0=27-q$.

(c) $18-5+x+5-8=18+x-8=18-8+x=10+x$.

¿Crees que el profesor le pondrá un bien?

dan lugar a trabajar la justificación de las igualdades. Aquí se ponen en juego las reglas de cálculo para determinar si las simplificaciones están bien realizadas. Un error del tipo $d-3+10=d-13$ es una producción que pone de manifiesto "un modo de hacer" que tuvo su éxito en el dominio de la aritmética y que es necesario hacerlo consciente y rechazarlo para dar lugar al nuevo cálculo. En aritmética es relativamente frecuente que los alumnos realicen, de izquierda a derecha, las secuencias de sumas y restas indicadas. En este caso, la primera sucesión de cuentas que pueden reconocer es $3+10$, ya que a $d$ no se puede restar 3 . Haber percibido esta situación fue un hecho muy importante en el grupo de discusión en el espacio colaborativo, pues permitió dar una interpretación a un hecho muy recurrente en las clases.

Entre los dos tipos de problemas descriptos anteriormente, contextualizados y descontextualizados, se tiene que dar un proceso donde hay que lograr que las expresiones algebraicas se tomen como objeto de estudio. Para ello hay que crear lazos entre la equivalencia de las expresiones $x-9+7$ y $x-2$, apoyada por el contexto, por ejemplo "figuritas perdidas y ganadas" con el cálculo $x-9+7=x-2$, apoyado en las reglas "restar 9 y sumar 7 es lo mismo que restar 2 ". El último problema pone en discusión el modo en que se realizan esas cuentas, permitiendo poner en evidencia la diferencia entre los cálculos aritmético y algebraico. El paso de la actividad aritmética a la algebraica supone transitar de un cálculo entre números sin determinación (números absolutos) a un cálculo en el que hay que tener en cuenta la condición de sumandos y sustraendos de los números.

A partir de problemas de este tipo se favorece la circulación en la clase de los siguientes conocimientos: "la respuesta a un problema puede ser una expresión o una relación (no solamente un número)"; "si no se conoce un dato, se usa una letra"; "se representan situaciones armando expresiones con números y letras"; "hay distintas expresiones para representar lo mismo" y "simplificar expresiones sin contexto, usando reglas de cálculo para la suma como las siguientes":

1) "restar 9 y sumar 2 es lo mismo que restar 7 ",

2) "restar 5 y luego sumar 7 es lo mismo que sumar 7 y luego restar 5",

3) si en una expresión se suma y resta un mismo número, se tachan los dos y se vuelve a escribir toda la expresión sin esos términos. 
$\S 3$. Acerca de la resta como diferencia y construcción de las reglas de cálculo

para restar

Para abordar la resta será importante seguir ahondando en las rupturas necesarias entre lo aritmético y lo algebraico. Así, la concepción de número asociada a las magnitudes y la de resta vinculada a la acción de quitar, son ideas que obstaculizan la construcción del número negativo (Cid, 2000), dificultando su manipulación de forma aislada y su aceptación como resultado de una diferencia. Esto encuentra su explicación en que el cero absoluto que se identifica con la ausencia de cantidad de magnitud, la nada, el vacío, dificulta pensar en una resta en la que el sustraendo es mayor que el minuendo, condicionado por la existencia de medidas que son "menos que nada". Será necesario entonces ampliar la concepción de resta como sustracción a una concepción de resta como diferencia y abandonar la concepción de número ligada al resultado de una medida de una cantidad de magnitud para concebir a un número como resultado de una diferencia.

Frente al tratamiento que se propone para introducir la diferencia nos encontramos, en los espacios de estudio compartidos entre docentes e investigadores, con un entramado de los conceptos comparación, diferencia, resta y presentación de número negativo, cuyo desentrañado fue un trabajo conjunto. Por un lado, tuvimos que comprender que asociar la diferencia con cuantificar una comparación amplía la concepción de la misma y que, asociar la diferencia con una resta permite ampliar las reglas de cálculos. Además, comprender que ambos asuntos se enlazan para presentar en la clase el número negativo como resultado de una diferencia, el cual indica que el minuendo es menor que el sustraendo. Por otro lado, advertimos que hay conocimientos matemáticos necesarios para avanzar en comprensión, que posiblemente no aparezcan como ideas de los estudiantes a partir de las actividades que ellos realicen, y entonces fue necesario discutir la ocasión de ser explicitados. La idea de la diferencia a partir de comparar es una cuestión que surge de la reflexión de las actividades a resolver, sin embargo, asociar la diferencia con la operación restar, podría ser un objeto a declarar por parte del docente. Algo similar ocurre con la escritura de los paréntesis en una expresión para indicar una operación de una operación, como por ejemplo la resta de una suma o la resta de una resta.

En un contexto algebraico, comparar expresiones como $a+6$ y $b-2$ y expresar en cuánto una es mayor/menor que la otra, conduce a encontrar la diferencia y gestionar expresiones del tipo: $a+6-(b-2)$, a la vez que permite concebir la resta sin la restricción de que el minuendo sea mayor que el sustraendo. Así, la resta entre expresiones surge para hallar la diferencia entre ellas y lo que da lugar a ese proceso es la comparación de expresiones. El significado de la resta 
como diferencia ${ }^{4}$, está relacionado con la acción de comparar y de cuantificar esa comparación, la cual será positiva o negativa según que el minuendo sea mayor o menor que el sustraendo. Además, reinterpretar la resta como diferencia dando la posibilidad a una diferencia negativa, contribuye a dar sentido al número negativo, ligado al álgebra, como un número con la propiedad de ser menor que cero. Así, el número -3 surgirá, por ejemplo, de la diferencia entre las expresiones $x-4$ y $x-1$. El resultado de $x-4-(x-1)$ es -3 , un número 3 con un signo "-", que indica dos cosas: “ 3 ", revela de cuánto es la diferencia entre esas expresiones y el signo "-" del número informa que la segunda expresión es mayor que la primera (Martinez y Issa Nuñez, 2019).

En principio se deberán ampliar las reglas de cálculo y pasar de efectuar operaciones entre sumandos y sustraendos a efectuar operaciones de operaciones, lo que da lugar a nuevos significados de los signos $+\mathrm{y}-$. Es interesante compartir aquí, que antes de la aceptación de los números negativos como números aislados, los matemáticos realizaban cálculos sólo con números positivos y utilizaban distintas propiedades para la resta como la siguiente:

$$
\text { si } b<c \text { entonces } a-(b-c)=c-(b-a)=(a-b)+c \text {. }
$$

Esta propiedad permite realizar restas entre números naturales, aunque los cálculos intermedios sean negativos, como se ve en el siguiente ejemplo: $5-(9-12)=$ $12-(9-5)$. Así, pensar en la resta de la resta o la resta de una suma ha sido una cuestión constitutiva de este conocimiento a lo largo de la historia y la técnica de supresión de paréntesis no siempre estuvo presente. Entonces, para trabajar la resta entre sumandos y sustraendos será necesario evolucionar en los significados de los signos $+\mathrm{y}-$, del conocido operador binario (resta entre números) a uno nuevo, el operador unario (el menos delante del paréntesis indica un cambio en los sumandos y sustraendos que intervienen dentro del paréntesis). Esto significa, en la práctica, desarrollar un cálculo con términos, que a su vez son operaciones indicadas, para reducirlas a sumas de sumandos y sustraendos. Por ejemplo, ante situaciones como $4-(b-7)$, para encontrar la expresión simplificada, la idea que funciona es la de restar una resta y aplicar la regla de cálculo "restar $(b-7)$ es lo mismo que restar $b$ y sumar 7 ". Este abordaje, en el que emerge la regla que permite suprimir paréntesis, se constituirá en el soporte en el que se apoyará la justificación de la resta de números enteros.

3.1. Actividades para la concepción de diferencia. Veamos, en particular, un tipo de problema contextualizado que permite asociar la diferencia con la comparación y su cuantificación.

\footnotetext{
${ }^{4}$ La resta como diferencia es el resultado de una comparación entre los cardinales de dos conjuntos en un orden establecido que informa en cuánto es mayor o menor uno que otro. Por ejemplo, si $A$ tiene $x$ y $B$ tiene $y$, la diferencia entre lo que tiene $A$ y lo que tiene $B$ es $x-y$, es decir que $A$ tiene $x-y$ elementos más/menos que $B$.
} 
Al empezar la semana, María, Adrián y Luisa tienen el mismo dinero en su alcancía. Entre lunes y jueves gastan o reciben las siguientes cantidades:

\begin{tabular}{|c|c|c|}
\hline María & Adrián & Luisa \\
\hline Recibe \$10 & Gasta \$5 & Recibe \$10 \\
\hline Gasta \$5 & Gasta \$10 & Recibe \$5 \\
\hline Gasta \$15 & Gasta \$15 & Recibe \$15 \\
\hline & Recibe \$30 & Gasta \$35 \\
\hline
\end{tabular}

(a) ¿Quién tiene más dinero? ¿Quién tiene menos? ¿En cuánto se diferencia de los demás el dinero que tiene cada uno?

Las primeras preguntas que se refieren a quién tiene más o menos, remiten a una comparación. La situación contextualizada permite dar respuestas a partir de las acciones de "recibir" y "gastar". Así, el que tiene más es Adrián pues recibe y gasta lo mismo. María es la que tiene menos dinero ya que tiene 10 menos que al principio mientras que Luisa tiene 5 menos que al principio. La última pregunta conlleva a una cuantificación de la diferencia, la cual surge en forma inmediata entre Adrián y las demás, pues él queda con el mismo dinero que al inicio. La cuestión es entre María y Luisa, que remite a buscar la diferencia entre la cantidad de dinero de una y de otra, esto es, $x-10$ y $x-5$ y sin necesidad de realizar el cálculo escrito se puede concluir que esa diferencia es de 5 .

Este problema admite una resolución desde el contexto mismo de la situación, lo importante aquí es reflexionar, sobre el accionar más allá de la respuesta. Es decir, destacar que para hallar una diferencia, por ejemplo, entre lo que tienen dos personas, está presente la comparación pues se evalúa cuánto más o cuánto menos tiene una en relación a la otra.

3.2. Actividades para construir las reglas de cálculo para restar. En un principio las tareas que se proponen para construir estas reglas de cálculo, se apoyan en conocimientos aritméticos. Para realizar operaciones entre dos números se recurre a un cálculo auxiliar donde los números redondos facilitan las cuentas, así sumar 100 y restar 1, es más fácil que sumar 99. Un ejemplo de este tipo de actividades es el siguiente:

Cuando se calcula mentalmente se procura buscar la forma más sencilla posible de efectuar las operaciones. ¿Cómo lo haces con las siguientes operaciones?
a) $678+99$
b) $157-99$
c) $601-103$

Luego, el registro de ese cálculo auxiliar pondrá en escena el paréntesis. El cálculo de las operaciones indicadas dará lugar a nuevas reglas de cálculo que permitirán abordar operaciones de operaciones. Para tal fin se proponen tareas del tipo: 
Coloca los signos $+y-q u e$ faltan en las siguientes igualdades:

$$
\begin{aligned}
& 765-(100-1)=765-99=765 \_100 \_1 . \\
& 80-(30-1)=80-29=80 \_30 \_1 . \\
& 141-(100+2)=141-102=141 \_100 \_2 .
\end{aligned}
$$

La actividad matemática involucrada en esta última tarea, se relaciona con la primera en el sentido que en $765-(100-1)$ se visualiza la escritura con paréntesis; luego se vincula con 765 - 99, que proviene de aplicar la prioridad que establece el paréntesis y a su vez es una expresión en la que convendría usar la estrategia de los números redondos, y finalmente completar $765 \_100 \_1$ da lugar a registrar las estrategias puestas en juego en los cálculos mentales realizados en la actividad anterior. Si se efectúa la cuenta resolviendo primero el paréntesis, se pierde el número redondo. La búsqueda de economía de los cálculos lleva entonces a explorar una regularidad entre $765-(100-1)$ y $765-100+1$. Esas relaciones que vinculan una operación de operación con la suma de sumandos y sustraendos van a permitir institucionalizar las reglas de cálculo que darán lugar a suprimir el paréntesis.

Por otro lado, la comparación de expresiones permitirá, como veremos más adelante, determinar si una diferencia entre expresiones es positiva o negativa, dando lugar a que emerja un sentido del número negativo. Será necesario entonces poder determinar si una expresión algebraica es menor o mayor que otra en actividades descontextualizadas, donde las acciones que comandan las decisiones sean las operaciones de "sumar" o "restar" en lugar de las del tipo "recibir" o "gastar", como vimos en "Actividades para la concepción de la diferencia". El siguiente ejemplo tiene ese objetivo:

Compara las siguientes expresiones, diciendo cuál de ellas es menor o mayor que la otra:
a) $x+1$ $x-10$.
b) $p-7 \_p-3$.
c) $2 a+5 \quad 3 a+12$.
d) $25-z \_25-2 z$.
e) $3 n+5 \_2 n+30$.

Para analizar qué expresión es menor/mayor que otra, una técnica posible es comparar los términos de cada expresión. Por ejemplo, en el ítem c) ${ }^{5}, 2 a+5$ es

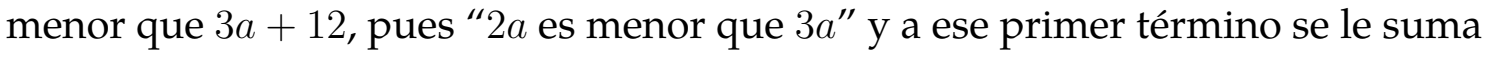
algo menor que lo que se suma a la segunda expresión. Aquí surgen técnicas de comparación de expresiones, analizando término a término. Con el ítem e) se pone

\footnotetext{
${ }^{5}$ Tengamos presente que las letras de estas expresiones tienen como dominio los números naturales.
} 
en evidencia que a veces no se puede decidir cuál expresión es mayor, porque depende del valor de la variable.

El siguiente problema tiene por objetivo asociar la diferencia con el cálculo de una resta y el uso de paréntesis.

Carlos tiene 6 canicas más que Javier y Enrique 10 canicas menos que Marcos. Si sabemos que Carlos tiene más canicas que Enrique, ¿cuántas más tiene?

\begin{tabular}{|l|l|}
\hline$N^{o}$ de canicas de Javier & \\
\hline$N^{o}$ de canicas de Carlos & \\
\hline$N^{o}$ de canicas de Marcos & \\
\hline$N^{o}$ de canicas de Enrique & \\
\hline Diferencia entre el $n^{o}$ de canicas de Javier y Marcos & \\
\hline Diferencia entre el $n^{o}$ de canicas de Carlos y Enrique & \\
\hline
\end{tabular}

La discusión y análisis, al interior del grupo colaborativo, de las posibles resoluciones del problema, nos llevó a reflexionar sobre las intervenciones del docente para abordar códigos de escrituras (registro de la diferencia como una resta y la ocasión de escribir el paréntesis) como un conocimiento nuevo.

Para establecer la diferencia entre el número de canicas de Javier y Marcos, siendo $x$ : cantidad de canicas que tiene Javier e $y$ : cantidad de canicas de Marcos, las expresiones para las que hay que hallar su diferencia tienen variables diferentes, $x$ e $y$, por lo tanto, la técnica de comparación "término a término", descrita anteriormente, se vuelve insuficiente. Advertimos que se estaba poniendo en juego el registro de la diferencia entre expresiones y su cálculo. Esto trajo a discusión cómo y cuándo explicitar en la clase que ese registro se corresponde con una resta y destacar la orientación de la misma, es decir aclarar que: "para indicar la diferencia entre $x$ e $y$ se escribe $x-y$, para registrar la diferencia entre $y$ y $x$ se escribe $y-x^{\prime \prime}$.

Por otro lado, al anticipar la resolución de los alumnos para completar la fila que solicita la diferencia entre Carlos: $x+6$ y Enrique: $y-10$, pusimos el foco en el uso de paréntesis, pues en esta resolución es necesario proponer expresiones en las que se involucra la escritura de este símbolo. Observemos que en la aritmética escolar el paréntesis en las expresiones viene dado, la experiencia que tienen los alumnos es entonces, interpretar la información que brinda ese símbolo en relación a la prioridad de las operaciones. En cambio, en este problema, se pone en juego un código de escritura que responde a una "norma matemática". Se abre así un espacio para explicitar el uso del paréntesis para expresar operaciones de operaciones, en particular la resta de una suma o la resta de una resta.

3.3. Actividad para que emerja el número negativo. El siguiente problema, presentado en forma descontextualizada, da lugar a una nueva concepción de número junto al significado predicativo de los signos $+\mathrm{y}-$. 
Calcula la diferencia entre las siguientes expresiones algebraicas:
a) $7 p+3 q$
$2 p-2 q$
b) $4 t-6-15$
$4 t-3-2$
c) $21-2 m+3-23+5 m$
$10 m-30+5 m+25$

Este tipo de problema exige poner en funcionamiento los conocimientos anteriores. Por ejemplo, en b) se podría calcular la diferencia entre $4 t-6-15$ y $4 t-3-2$ comparando $4 t-21$ con $4 t-5$. Usando la técnica de comparación término a término se puede decir que las expresiones difieren en 16 y cuál de ellas es mayor. Si bien la respuesta es -16 , con los conocimientos disponibles por parte de los alumnos, esta respuesta es poco factible que surja en la clase. Frente a esta situación se abre un espacio fértil para que el docente, a partir de sus intervenciones, pueda ir enlazando la respuesta probable del alumno, 16, con la presentación del número negativo, -16 , interpretado como el resultado de una diferencia en la que el minuendo es menor que el sustraendo. ¿Cuáles son esos conocimientos que se deberían enlazar?

Para calcular la diferencia entre $4 t-6-15$ y $4 t-3-2$, también se podría realizar: $4 t-6-15-(4 t-3-2)$, que implica recuperar la asociación de la diferencia con la resta y la aplicación del paréntesis. Esto da lugar a las técnicas de cálculos, en un principio las de la suma, por ejemplo "restar 6 y restar 15 es lo mismo que restar 21 ", obteniendo $4 t-21-(4 t-5)$; luego las reglas de cálculo de la resta de una resta: "restar $4 t-5$ es lo mismo que restar $4 t$ y sumar 5 " quedando: $4 t-21-4 t+5 \mathrm{y}$ por la técnica de simplificación se "tachan" $4 t \mathrm{y}-4 t$, finalmente se obtiene $-21+5$. Con este último cálculo surge lo nuevo.

Entonces, hay que asociar con el resultado de este cálculo:

$$
4 t-6-15-(4 t-3-2)=\cdots=-21+5=-16,
$$

que las expresiones $4 t-6-15$ y $4 t-3-2$ difieren en 16 y que la primera es menor que la segunda. Así, el - 16 surge de interpretar por un lado el "16" como la cantidad en la que difieren las expresiones y por otro lado ese signo "-" delante del número como la cualidad del número que indica que esa diferencia es negativa, pues el minuendo es menor que el sustraendo. Se comienza a aceptar a estos nuevos números con signo como resultado de una diferencia. Así, se establece que el resultado del cálculo de la diferencia entre expresiones indica en cuánto una de ellas sobrepasa a la otra y el signo muestra cuál es la mayor. Además, se puede establecer el cálculo de una diferencia como una técnica para comparar los términos de una diferencia, según su resultado sea positivo o negativo, da información de cuál término es mayor o menor.

Los problemas que presentamos en esta sección, favorecen la circulación en la clase de los siguientes conocimientos de los alumnos: "para hallar una diferencia se puede comparar y decir cuánto más/menos tiene una expresión en relación a otra"; "hay expresiones que son mayores/menores que otras y hay otras que no se 
pueden comparar"; "para hacer las cuentas más fáciles se usan números redondos"; "para hallar la diferencia entre expresiones que no se pueden comparar, se hace una resta y se tiene que usar paréntesis para la expresión que está restando"; "la diferencia puede ser positiva o negativa y eso depende de que la primera expresión sea mayor o menor que la segunda en la resta"; "si la diferencia es negativa se escribe el número con el signo - "; "Si una cuenta tiene paréntesis se hace primero la cuenta del paréntesis o se resuelve con las siguientes reglas de cálculos para restar una suma o restar una resta":

- Restar $a+b$ es lo mismo que restar a y restar $b$.

- Restar a - b es lo mismo que restar a y sumar $b$.

- Sumar a + b es lo mismo que sumar a y sumar b.

- Sumar $a$ - b es lo mismo que sumar a y restar $b$.

\section{§4. Acerca de la construcción de las reglas de cálculo para multiplicar}

Para abordar el producto de sumandos y sustraendos se sigue profundizando en el funcionamiento algebraico de algunos objetos matemáticos, contrapuesto al aritmético. Para ello la representación simbólica y el trabajo con expresiones aditivo-multiplicativas son asuntos que están presentes. El dominio y manipulación de programas de cálculos del tipo $a(x \pm b)-c(x \pm d)$, siendo $a, b, c$ y $d$ números naturales, pone de manifiesto la importancia de la propiedad distributiva en el cálculo algebraico y permite establecer las reglas del producto de sumandos y sustraendos. Además, la escritura convencional del producto en el álgebra requiere dejar de lado el uso de la cruz: $3 \times a$ o $4 \times 5$ para indicar una multiplicación, del mismo modo que analizar cuándo se hace necesario usar paréntesis en una expresión. Asumir que en $a b$ y que en $a(x+y)$ hay una operación que no se escribe y que hay nuevas reglas que involucra la distributiva son parte de los conocimientos necesarios para avanzar en el cálculo algebraico.

Las reflexiones y discusiones en los espacios de estudio compartidos entre docentes e investigadores, nos llevaron a comprender el rol que cumple el trabajo con este tipo de expresiones en una nueva ruptura con el cálculo aritmético. Las secuencias de sumas y restas, frecuentes en aritmética, son realizadas por los alumnos de izquierda a derecha, este tipo de procedimiento es el que hay que hacer evolucionar. En este sentido (Cid, 2015) plantea:

"La jerarquía de las operaciones que establecen las reglas del cálculo algebraico rompe con la técnica aritmética de efectuar las operaciones de izquierda a derecha, imponiendo una lectura de toda la expresión algebraica para efectuar en primer lugar aquellas operaciones que tienen prioridad, independientemente del lugar que ocupen en la expresión" (p. 286). 
Esta comprensión nos permitió interpretar que un procedimiento anclado todavía en un cálculo aritmético, se manifestaría en errores como se observa en el siguiente ejemplo:

$$
5-3(4+x)=2(4+x)=2.4+2 x=8+2 x=10 x
$$

Por otro lado, resignificamos conocimientos implicados cuando se aplica la propiedad distributiva del producto con la suma de números enteros. Habitualmente, en la enseñanza del nivel secundario, la resolución de este tipo de expresiones se trabaja después de tener definidos los números enteros y explicitada la regla de los signos. Así, la justificación que $9-5(m-4)$ es $9-5 m+20$ deviene de concebir la expresión $9-5(m-4)$ como $9+(-5)(m+(-4))$, donde el signo + no está presente en forma explícita y esta ausencia frecuentemente pasa inadvertida en la enseñanza.

Entonces, debimos reconstruir un camino retomando los conocimientos construidos anteriormente que permita, a partir de la creación y manipulación de programas de cálculos aritméticos y del trabajo con la notación incompleta ${ }^{6}$, justificar que $9-5(m-4)$ es igual a $9-5 m+20$. La justificación se sostiene por los cálculos de primero distribuir el cinco y luego concebir la operación resultante como la resta de una resta. El estudio del funcionamiento de estas expresiones habilita la gestión de una operación entre paréntesis multiplicada por un sumando o un sustraendo. Así, trabajando primero con la propiedad distributiva y operación de operaciones (por ejemplo, resta de una resta, resta de una suma), se logran condiciones para apuntalar una justificación de la conocida "regla de los signos" ("menos por menos es más", "más por menos es menos", etc.).

A continuación mostramos el proceso que dará lugar a gestionar expresiones del tipo $a(x \pm b)-c(x \pm d)$, a través de un contexto geométrico que involucra el cálculo y la comparación de áreas de rectángulos ${ }^{7}$.

\subsection{Actividades para construir las reglas de cálculo para multiplicar. En una} primera instancia el trabajo se centra en la representación simbólica de expresiones de la forma $a x$ y $a(x+b)$, es decir en las distintas escrituras para simbolizar la operación de multiplicación y el uso del paréntesis. Para ello, en este primer problema, se solicita expresar las distintas áreas de los rectángulos y encontrar la diferencia entre las mismas, diferencia que se puede hallar sin necesidad de los cálculos.

\footnotetext{
${ }^{6}$ La diferencia entre la notación algebraica completa y la incompleta es que en la primera figuran los signos de suma y producto entendidos como indicadores de operaciones binarias entre términos, mientras que en la incompleta estos símbolos se suprimen (Cid, 2015, p.212). Por ejemplo: notación completa: $(-3)+$ $(+5)-(+7)$; notación incompleta: $-3+5-7$.

${ }^{7} \mathrm{Si}$ bien los números representan medidas de longitud y, en principio, podrían tomar valores positivos no enteros, las letras de las expresiones siguen teniendo dominio en el conjunto de los números naturales.
} 
Como ya saben, la fórmula del área de un rectángulo es $A=b a$, donde $b$ es la longitud de uno de los lados (base) y a la longitud del otro lado (altura).

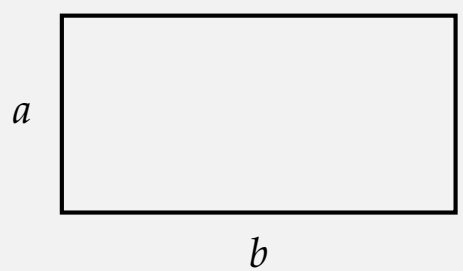

i) Si nos dicen que $a=3 \mathrm{~cm}$, ¿cómo expresaremos el área de ese rectángulo?

ii) Si ahora te dicen que en ese rectángulo el lado baumenta $2 \mathrm{~cm}$, haz un dibujo del nuevo rectángulo. ¿Cuál será ahora la longitud de sus lados? ¿Cuánto habrá aumentado su área?

El enunciado del problema expresa "el área de un rectángulo es $A=b a$ ", esto dará lugar a la discusión acerca de cómo simbolizar el producto, a partir de analizar las distintas expresiones que puedan surgir para expresar el área del rectángulo: $3 \times b, 3 . b, 3 b$ o $b \times 3, b .3, b 3$. En el inciso ii) el lado desconocido sufre modificaciones y el conocido no cambia. Para resolver lo solicitado, en primer lugar, se tiene que indicar la medida de un lado, que no es un número ni una letra, ahora es una expresión, en este caso " $b+2$ ". Para comparar las áreas de los rectángulos no es necesario realizar la diferencia de las expresiones, ya que en este caso se puede resolver desde el gráfico (Colipe, Zambrano, y Ruiz, 2018), como lo muestra el rectángulo de la derecha en la Figura 1:
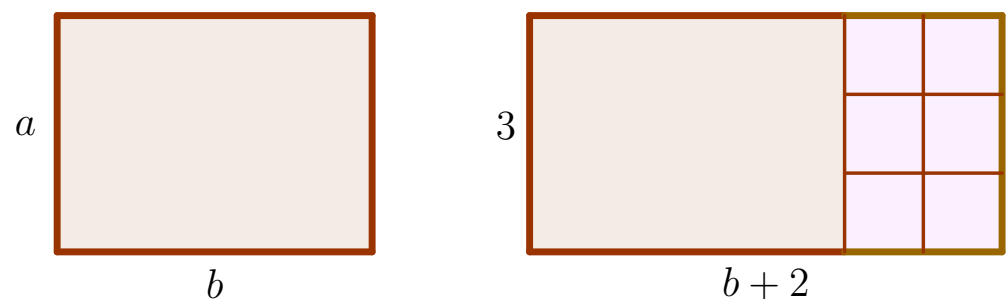

FIgURA 1. Rectángulo original y rectángulo ampliado

El contexto geométrico y la comparación de áreas en las que sólo varía un lado, permite encontrar y justificar que la diferencia entre las expresiones corresponde a un número. Percibir que la ampliación corresponde a un rectángulo de lados 2 y 3 (ver Figura 1) es lo que permite asegurar que el incremento es de 6. El trabajo algebraico encuentra su apoyo en lo geométrico, pues el dibujo facilita encontrar que la diferencia entre las áreas de los rectángulos (original y ampliado) es 6 y en esa certeza se gestiona la construcción de nuevas expresiones en las que el paréntesis empieza a tener protagonismo.

En el grupo colaborativo, un trabajo de análisis fue imaginar diálogos entre posibles producciones de los estudiantes en distintos contextos (geométrico y 
algebraico). Expresar al área del rectángulo ampliado llevará a la cuestión de cómo construir expresiones en las que el uso del paréntesis es objeto de análisis. Así, hipotetizamos distintas expresiones que podrían surgir según lo que se ponga en juego. Por ejemplo: si usan la fórmula del área para un rectángulo de lados 3 y $b+2$ surgirán expresiones como: $b+2.3$ o $3 b+2$ o $3(b+2)$ o $(b+2) 3$; si, apoyados en el dibujo del rectángulo ampliado de la Figura 1, descomponen en dos áreas, una es $3 b$ o $b 3$ y la otra es 6 , aparecerán expresiones como: $3 b+6$ o $b 3+6$ y si construyen una expresión 6 unidades más grande que $3 b$ podrán escribir: $3 b+6$.

Analizar y establecer vínculos entre estas posibles expresiones da lugar a un trabajo acerca del uso oportuno de paréntesis y a establecer la equivalencia entre $3(b+2)$ o $(b+2) 3$ y $3 b+6$ o $b 3+6$. Y descartar expresiones tales como $b+2.3$ que es $b+6$ y $3 b+2$ porque no corresponden a expresiones " 6 unidades mayor que $3 b^{\prime \prime}$, área del rectángulo ampliado.

En un segundo problema, se trabaja con expresiones de la forma $a x$ y $b(x+c)$ con $a \neq b$, buscando la diferencia entre áreas de rectángulos, la cual no será un número, sino una expresión. El contexto geométrico no alcanza para dar una respuesta y se hace necesario avanzar en cálculos que dan lugar a recuperar el vínculo entre diferencia y resta.

En este caso, el rectángulo dado sufre aumento y disminución de sus lados en forma simultánea, lo que no permite anticipar si aumenta o disminuye el área del rectángulo modificado. Esto habilita a involucrarse con el cálculo de la diferencia. Sin embargo, depende de qué diferencia se calcule, se activa o no la propiedad distributiva entre sumandos. Las áreas a comparar son $4 x$ y $6(x-1)$, en caso que se busque la diferencia haciendo $6(x-1)-4 x$, si bien hay que distribuir, es la propiedad distributiva entre naturales. En caso que se realice la otra diferencia $4 x-6(x-1)$ exigiría un análisis como lo detallamos en el siguiente problema:

A partir de un rectángulo del que conocemos la longitud de un lado, 5 cm, construimos otros dos rectángulos: uno en el que aumentamos el lado conocido en $3 \mathrm{~cm}$ y el desconocido lo disminuimos en $3 \mathrm{~cm}, y$ otro en que disminuimos el lado conocido en $3 \mathrm{~cm}$ y aumentamos el lado desconocido en $3 \mathrm{~cm}$.

a) Haz un dibujo del primer rectángulo.

b) Haz un dibujo del segundo y tercer rectángulos.

c) Encuentra la diferencia entre las áreas de los dos últimos rectángulos. ¿Cuánto tiene que medir el lado desconocido para que las dos áreas sean iguales?

En la Figura 2 se muestran los dibujos de los tres rectángulos solicitados en la consigna. 


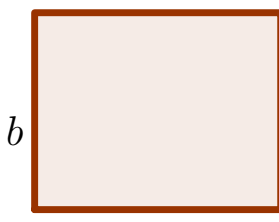

5 $b-3$

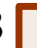

8

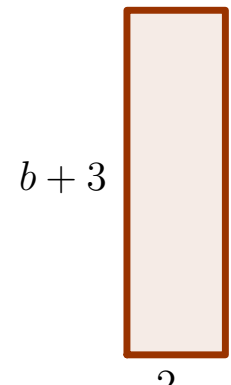

2

FIgURA 2. Rectángulos solicitados en la consigna

Este problema plantea tanto el aumento como la disminución de los lados de un rectángulo y propone estudiar las modificaciones de las áreas de los rectángulos resultantes. Aquí, se pone en juego comparar y hallar la diferencia entre expresiones del tipo $a(x \pm b)$ y $c(x \pm d)$ donde $a$ y $c$ son distintos, lo cual exige lidiar con cálculos como $a(x \pm b)-c(x \pm d)$, donde la propiedad distributiva tiene un rol fundamental.

Reconocemos la importancia de abordar este trabajo siendo conscientes que, de modo similar a lo que ocurre con las reglas para sumar, aquí también se pone en tensión un "hacer aritmético" que persiste y encuentra un límite en estas nuevas cuentas. La búsqueda de las diferencias entre las expresiones indicadas anteriormente, obliga a cálculos que deberán evolucionar de aplicar la propiedad distributiva entre números naturales (ya conocida), hacia una distributiva entre sumandos o sustraendos. Las reglas de cálculos para la suma y la resta, construidas anteriormente, se constituirán en el soporte para llevar a cabo este proceso.

Así, por ejemplo, para resolver el inciso c) y si consideramos que $A_{2}=8(b-3)$ y $A_{3}=2(b+3)$ representan las áreas de los rectángulos modificados (ver Figura 2 ), la diferencia entre las mismas implica cálculos que se justifican en las reglas aprendidas, como se muestra a continuación:

$$
A_{2}-A_{3}=8(b-3)-2(b+3),
$$

si se aplica la propiedad distributiva de números naturales (se distribuye el 8 y el $2)$, se obtiene $8 b-24-(2 b+6)$. Por la regla de cálculo para la resta: restar $2 b+6$ es lo mismo que restar $2 b$ y restar 6 , quedando entonces $8 b-24-2 b-6$. Por la regla de cálculo para la suma: restar primero 24 y luego restar $2 b$ es lo mismo que restar primero $2 b$ y luego restar 24 , queda $8 b-2 b-24-6$. Finalmente, por regla de cálculo para la suma, restar 24 y restar 6 es lo mismo que restar 30 quedando $6 b-30$.

Es importante vincular las expresiones donde está presente el producto de un sustraendo por una suma o diferencia como " $8(b-3)-2(b+3)$ " y las que se reducen a sumas de sumandos y sustraendos como " $8 b-24-2 b-6$ ". Así, afirmándose en conocimientos disponibles, como lo son la distributiva entre naturales y la resta de una resta, da lugar a una nueva técnica que permitirá resolver el producto de un sumando o sustraendo por una suma o diferencia. 
A partir del trabajo con las actividades anteriores, se favorece en clase la circulación de los siguientes conocimientos: "para escribir el producto no se coloca nada, salvo que sean dos números, y en ese caso se usa el punto"; "para hallar la diferencia entre expresiones se puede compararlas y decir en cuánto más grande o más chica es una en relación a la otra o se calcula una resta entre ellas"; "si a una expresión se le suma o se le resta un número, la expresión será mayor o menor que la dada"; "si hay que multiplicar un número con una suma o una resta se tiene que usar paréntesis". Y surgen las reglas de cálculo del tipo:

- Restar $2(a+b)$ es lo mismo que restar $2 a$ y restar $2 b$.

- Restar 2(a-b) es lo mismo que restar $2 a$ y sumar $2 b$.

- Sumar $2(a+b)$ es lo mismo que sumar $2 a$ y sumar $2 b$.

- Sumar $2(a-b)$ es lo mismo que sumar $2 a$ y restar $2 b$.

Hasta aquí hemos visto un recorrido en el que surgen las reglas de cálculo para la suma, resta y multiplicación de sumandos y sustraendos apoyados en los conocimientos de los números naturales, donde las expresiones algebraicas tuvieron un rol fundamental. Este recorrido está atravesado por rupturas entre concepciones y modos de calcular que viven en la aritmética y que evolucionan a nuevas concepciones y funcionamientos en el álgebra.

La estructura aditivo-multiplicativa de sumandos y sustraendos que acabamos de desarrollar, se convertirá en el nuevo soporte para apuntalar las reglas de cálculos de suma, resta y multiplicación de los números enteros. Así, la regla que se usa en $8-(0-5)$ que es restar $0-5$ es lo mismo que restar 0 y sumar 5 , va a permitir justificar que $8-(-5)=13$. De modo similar, la regla que está en juego en $0-5(0-3)$ que es restar $5(0-3)$ es lo mismo que restar 5.0 y sumar 5.3 , permitirá justificar que $-5(-3)=15$.

Es decir, una vez que "los números con signos" estén declarados como nuevos números: los enteros, la operatoria de éstos encuentra su justificación en las reglas de cálculos de sumandos y sustraendos, a partir de asociar un número positivo con un sumando y un número negativo con un sustraendo.

\section{$\S 5$. A modo de cierre}

Como planteamos en la introducción de este artículo, los espacios de trabajo colectivos entre docentes e investigadores, constituyen un escenario favorable para discutir con los docentes las cuestiones fundamentales que se ponen en juego en secuencias de enseñanza más que para difundir los productos acabados de una investigación (Sadovsky y cols., 2016). En este caso el espacio colaborativo que formamos investigadores y profesores de escuela secundaria, toma una relevancia importante ya que nos permitió estudiar y profundizar una secuencia de enseñanza diseñada en una investigación que no es propia y analizar una posible gestión de 
las actividades para las clases. Como plantean (Perrin-Glorian y Baltar Bellemain, 2019), aún si en dicha investigación fue considerada la complejidad de la enseñanza en el aula, las condiciones para la difusión en las clases requieren ser estudiadas.

La propuesta que adaptamos, propone una ruptura epistemológica del quehacer algebraico frente al aritmético, alejándose de la concepción del álgebra entendida como aritmética generalizada, que es la más habitual en la escolaridad obligatoria. Toma como base conocimientos del cálculo aritmético que los estudiantes disponen y se propone, mediante rupturas, hacerlos evolucionar, hacia nuevos conocimientos propios del álgebra. Con el análisis precedente quisimos compartir algunos aspectos que nos resultaron relevantes en la comprensión conjunta (profesores e investigadores) de dicho proyecto de enseñanza que le da sentido a la operatoria y concepción de número entero. Reconstruimos un camino entre las ideas que se producen al resolver las tareas y los saberes a enseñar. En esta reconstrucción aparecen conocimientos contextualizados con cierta provisoriedad, tales como las reglas de cálculos para operar sumandos y sustraendos, los cuales no forman parte de la enseñanza habitual y aquí tienen un rol fundamental. Entonces, reconocer y apropiarse de estos conocimientos fue parte del trabajo que se realizó en esta experiencia.

\section{Bibliografía}

Barrio, E., y Petich, A. (2018). El rol de los significados de los signos "+" y "-" en la construcción de los números negativos. En Políticas y prácticas de producción y circulación de conocimientos. a 20 años del primer congreso de investigación educativa en la universidad nacional del comahue. Cipolletti, Argentina. (Conferencia llevada a cabo en el VII Congreso Nacional y V Internacional de Investigación Educativa)

Bednarz, N. (1997). Formación continua de los docentes de matemática: una necesaria consideración del contexto. Universidad de Quebec. (Trabajo mimeografiado)

Bednarz, N. (2013). Regarder ensemble autrement: ancrage et développement des recherches collaboratives en éducation au Québec. Recherche collaborative et pratique enseignante. Regarder ensemble autrement, 13-29.

Bednarz, N. (2015). La recherche collaborative. Carrefours de l'éducation, 1(39), 171184. Descargado de https://www.cairn.info/revue-carrefours -de-1-education-2015-1-page-171.htm

Chevallard, Y. (2005). La place des mathematiques vivantes dans l'enseignement secondaire. En C. Ducourtioux y P. Hennequin (Eds.), (p. 239-263). París, Francia: Publications de l'APMEP. Descargado de http: / / yves . cheval lard .free.fr/spip/spip/article.php3?id_article=48 
Cid, E. (2000). Obstáculos epistemológicos en la enseñanza de los números negativos. Boletín del Seminario Interuniversitario de Investigación en Didáctica de las Matemáticas, 10,1-15. Descargado de http : / / www •ugr.es / jgodino/ siidm/boletin10.htm

Cid, E. (2015). Obstáculos epistemológicos en la enseñanza de los números negativos (Tesis Doctoral no publicada). Universidad de Zaragoza, Zaragoza, España.

Cid, E., y Bolea, P. (2010). Diseño de un modelo epistemológico de referencia para introducir los números negativos en un entorno algebraico. Diffuser les mathématiques (et les autres savoirs) comme outils de connaissance et d'action, 575-594.

Cid, E., Muñoz-Escolano, J. M., y Ruiz-Munzón, N. (2020). La introducción de los REI en la formación de profesores: un ejemplo de REI-FP. Educaçao Matemática Pesquisa(22(4)), 646-660.

Cid, E., y Ruiz-Munzón, N. (2011). Actividades de estudio e investigación para introducir los números negativos en un entorno algebraico. En Un panorama de la TAD: Aportaciones de la teoría antropológica de lo didáctico (pp. 579-604). Barcelona, España.

Colipe, L., Zambrano, J., y Ruiz, M. E. (2018). Introducción al producto de números enteros a partir de multiplicar expresiones algebraicas. Un camino entre lo ideal y lo posible. En Políticas y prácticas de producción y circulación de conocimientos. A 20 años del primer congreso de investigación educativa en la Universidad Nacional del Comahue. Cipolletti, Argentina. (Conferencia llevada a cabo en el VII Congreso Nacional y V Internacional de Investigación Educativa)

Desgagné, S., Bednarz, N., Couture, L., C.and Poirier, y Lebuis, P. (2001). L'approche collaborative de recherche en éducation: un rapport nouveau à établir entre recherche et formation. Revue des sciences de l'éducation, 27(1), 33-64.

Detzel, P., y Martinez, R. (2017). Cálculo algebraico. Un escenario fértil para la construcción del sentido del número negativo. Novedades Educativas Centro de publicaciones educativas y material didáctico(315), 63-66.

Martinez, R., y Issa Nuñez, E. (2019). Enseñanza de los números negativos a través de la modelización algebraica: desafíos y ventajas. Novedades Educativas Centro de publicaciones educativas y material didáctico(344).

Perrin-Glorian, M. J. (2011). L'ingénierie didactique à l'interface de la recherche avec l'enseignement. Vers une ingénierie didactique de deuxième génération", editor $=$ Ç. Margolinas and M. Abboud-Blanchard and L. Bueno-Ravel and N. Douek and A. Fluckiger and P. Gibel and F. Wozniak. En En amont et en aval des ingénieries didactiques (pp. 57-78). Grenoble. Francia: La pensée sauvage Grenoble. 
Perrin-Glorian, M. J., y Baltar Bellemain, P. M. (2019). L'ingenierie didactique entre recherche et ressource pour l'enseignement et la formation des maitres. Caminhos da Educação Matemática, 9(1), 45-82. Descargado de https: / / aplicacoes.ifs.edu.br/periodicos/index.php/ caminhos_da_educacao_matematica/article/view/298

Sadovsky, P., Itzcovich, H., Quaranta, M. E., Becerril, M. M., y García, P. (2016). Tensiones y desafíos en la construcción de un trabajo colaborativo entre docentes e investigadores en didáctica de la matemática. Educación matemática, 28(3), 9-29.

Patricia Detzel

Departamento de Matemática, FaEA, Universidad Nacional del Comahue, Argentina.

(凶) pdetzel@gmail.com

María Elena Ruiz

Departamento de Matemática, FaEA, Universidad Nacional del Comahue, Argentina.

(凶) ruiz.melena@gmail.com

Lucas Colipe

Departamento de Matemática, FaEA, Universidad Nacional del Comahue, Argentina.

(凶) lukscolipe@gmail.com

Recibido: 4 de agosto de 2020.

Aceptado: 3 de marzo de 2021.

Publicado en línea: 5 de abril de 2021. 